\title{
NOTE
}

\section{Acinar cell carcinoma of the pancreas in coho salmon Oncorhynchus kisutch}

\author{
J. R. Heidel ${ }^{1, *}$, D. D. Rockey ${ }^{2, *}$, J. R. Duimstra ${ }^{1}$ \\ ${ }^{1}$ College of Veterinary Medicine and ${ }^{2}$ Department of Microbiology, Oregon State University, Corvallis, Oregon 97331, USA
}

\begin{abstract}
A coho salmon Oncorhynchus kisutch, collected during routine spawing procedures, had a large tumor adjacent to the liver and adherent to the pyloric ceca. Histologic and ultrastructural examination of the tumor revealed features consistent with a carcinoma of pancreatic acinar cell origin. This is the first reported pancreatic tumor in a salmonid.
\end{abstract}

Spontaneous neoplasms of the exocrine pancreas in fish are rare. Pancreatic adenomas have been reported in the European flounder Platichthys flesus (L.) and the greater redhorse Moxostoma valenciennesi Jordan (Fournie et al. 1988). An acinar cell adenocarcinoma has been described in a hybrid platyfish (Nigrelli \& Gordon 1951), and acinar cell carcinomas have been observed in an Atlantic tomcod Microgadus tomcod Walbaum, and a goldfish Carassius auratus (L.) (Fournie et al. 1988). In this report, we describe the gross, histologic, and ultrastructural features of a spontaneous malignant neoplasm of the exocrine pancreas in a coho salmon Oncorhynchus kisutch, which to our knowledge is the first published description of a pancreatic tumor in a salmonid.

An adult female coho salmon weighing ca $3.5 \mathrm{~kg}$ was harvested during routine spawning procedures at the Fall Creek Salmon Hatchery, Lincoln County, Oregon, USA, on November 21, 1990. Fall Creek is a small tributary of the Alsea River. The fish had an $8 \times 4 \times$ $3 \mathrm{~cm}$, smooth, lobulated, dark tan mass within the cranial-ventral aspect of the peritoneal cavity. This mass was firmly attached to the pyloric ceca, was separate from the liver, and compressed adjacent viscera (Fig. 1). It had several variably sized cysts which

\footnotetext{
- Addressee for correspondence

- Current address: National Institute of Allergy and Infectious Diseases, Rocky Mountain Laboratories, Hamilton, Montana 59840. USA
}

did not contain fluid. The mass was removed from the fish, placed on ice, and transported to the Veterinary Diagnostic Laboratory at Oregon State University where it was transferred into $10 \%$ neutral buffered formalin. Samples for histologic evaluation were embedded in either paraffin or glycol methacrylate and processed by routine techniques. Additional material was post-fixed in osmium tetroxide and embedded in epoxy resin for electron microscopy.

The mass consisted of uniform neoplastic cells arranged in sheets (Fig. 2). A modest fibrovascular stroma subdivided the sheets into poorly defined lobules. Acini were present; however, no distinct acinar lumina were seen by light microscopy. The neoplastic cells varied in shape from round to polyhedral to elongate. The cells possessed moderate amounts of cytoplasm, and many contained distinct eosinophilic cytoplasmic granules. The round to oval, variably sized nuclei had vesicular chromatin and many had prominent nucleoli. Mitotic figures were rarely observed. Individual cells were necrotic, represented by isolated karyorrhectic and pyknotic nuclei. Occasional multinucleated cells were present. Small ducts lined by cuboidal epithelial cells interrupted the sheets of neoplastic cells. The cysts were lined by one to multiple layers of the neoplastic cells, which were supported by a broad band of fibrous connective tissue. A thin fibrous capsule encircled the mass. Cords and islands of the neoplastic cells penetrated the connective tissue of cyst walls and capsule.

Electron microscopy revealed neoplastic acinar cells bordering acinar microlumina (Fig. 3). Junctional complexes were present along the apical portions of the cells. In other areas, distinctly polar cells were arranged in palisades along fragmented basement membranes. Numerous membrane bound secretory 


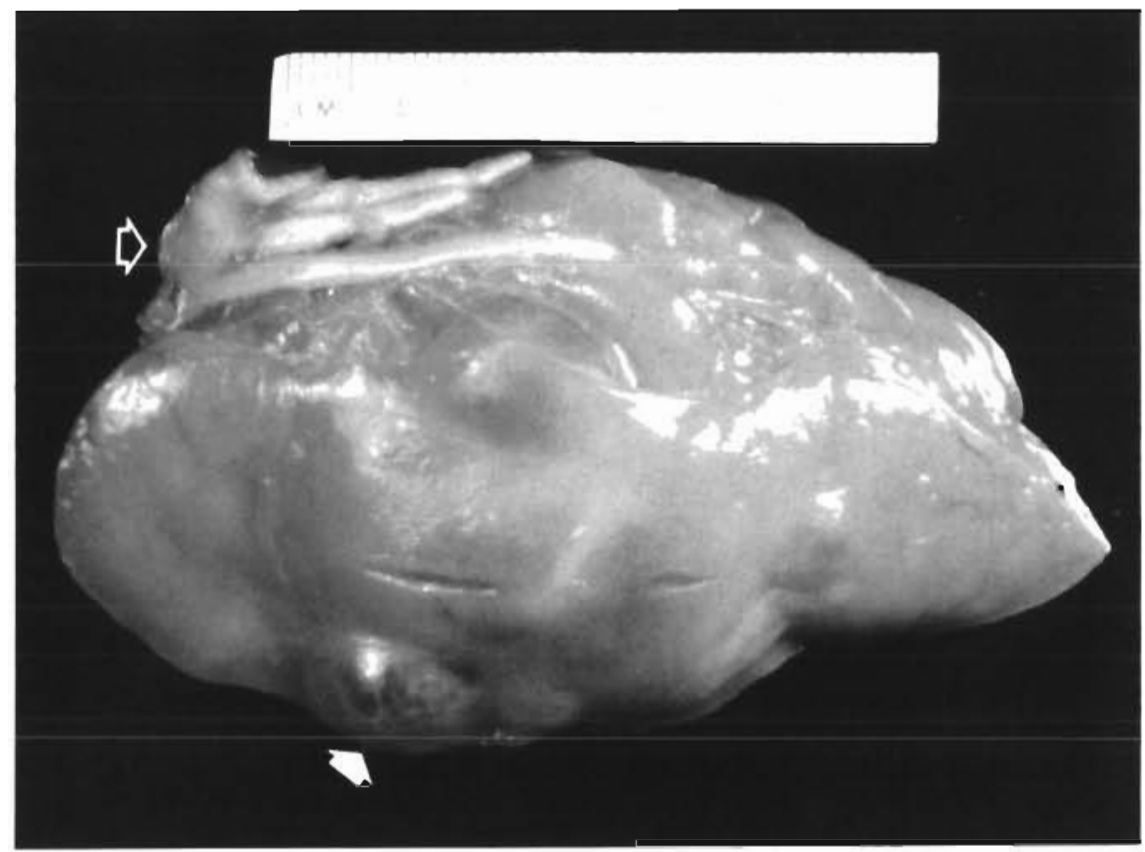

Fig. 1. Oncorhynchus kisutch. Pancreatic acinar cell carcinoma. Note cysts within body of tumor (solid arrow), and attached pyloric ceca (open arrow). Centimeter scale

granules, ranging in size from 400 to $1200 \mathrm{~nm}$ and varying in electron density, were within the apical cytoplasm. Situated between these granules and the nucleus were concentric rings of rough endoplasmic reticulum.
The histologic and ultrastructural features of the mass removed from this salmon are typical of those observed in tumors of the pancreas. The acini, although poorly formed and lined by polar cells, are characteristic histologic features of the exocrine pancreas, as are

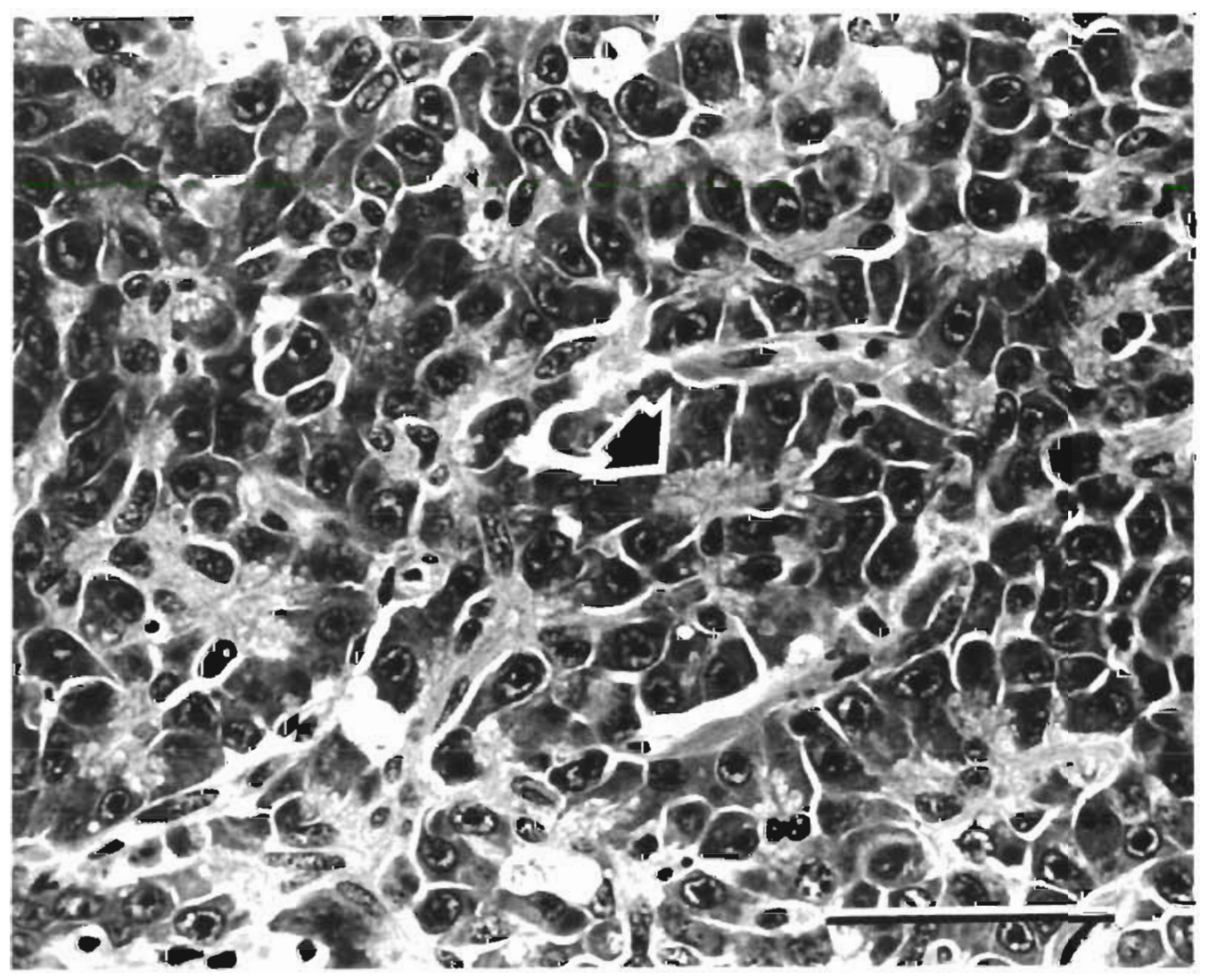

Fig. 2. Oncorhynchus kisutch. Photomicrograph of acinar cell carcinoma. Many acinar cells have distinct apical zymogen granules (arrow). Note cellular pleomorphism and distinct nucleoli. Bar = $50 \mu \mathrm{m}$ 
the abundant rough endoplasmic reticulum and apical zymogen granules observed in the electron micrographs (Jamieson 1988). The variation in electron density of the apical granules is associated with maturation of the organelle; maturation of the granules is accompanied by increased electron density.

The criteria for differentiating benign and malignant pancreatic tumors in fish are not well defined Although the tumor was circumscribed and possessed a fibrous capsule, and there was no gross evidence of metastasis within the fish, a diagnosis of carcinoma would be appropriate, based on the poor acinar differentiation, the cellular and nuclear pleomorphism, the prominent nucleoli, and the evidence of capsular invasion; invasiveness has been a criterion for malignancy of pancreatic neoplasms in fish (Thiyagarajah \& Grizzle 1986, Fournie et al. 1987). Although the mitotic activity was low, the degree of mitotic activity is variable amongst malignant pancreatic tumors (di Sant'Agnese 1991). As the tumor was separate from the liver, and as it was tightly bound to adjacent pyloric ceca, it is presumed that it arose from extra-hepatic pancreatic tissue, as has been seen in pancreatic tumors of the flounder and guppy (Fournie et al. 1988). However, the liver from this fish was not examined histologically.
This fish was moribund when harvested; its eggs were abnormal in appearance and considered unsuitable for use in fish propagation. Whether these signs were related to the presence of the tumor is not known. Unfortunately, no additional tissues from the fish were recovered for histologic evaluation, hence the presence of other lesions or evidence of tumor metastasis could not be verified. As this fish had been selected as part of a bacterial kidney disease monitoring project, a kidney sample was collected for identification of Renibacterium salmoninarum by ELISA (Rockey et al. 1991); bacterial antigen was not detected.

Spontaneous acinar cell carcinomas are rare not only in fish, but also in other animals (Popp 1990) and humans (Webb 1977). Little is known about the development of pancreatic tumors. In dogs and cats, most exocrine pancreatic carcinomas occur in older animals (Priester 1974). Although this spawning salmon would be in the older age group for this species, the small number of pancreatic tumors currently reported in fish, and the difficulties in accurately establishing the age of fish, would make correlations of age and tumor incidence tenuous. Exocrine pancreatic neoplasms have been experimentally induced in several rodent species (Scarpelli et al. 1984). Both benign

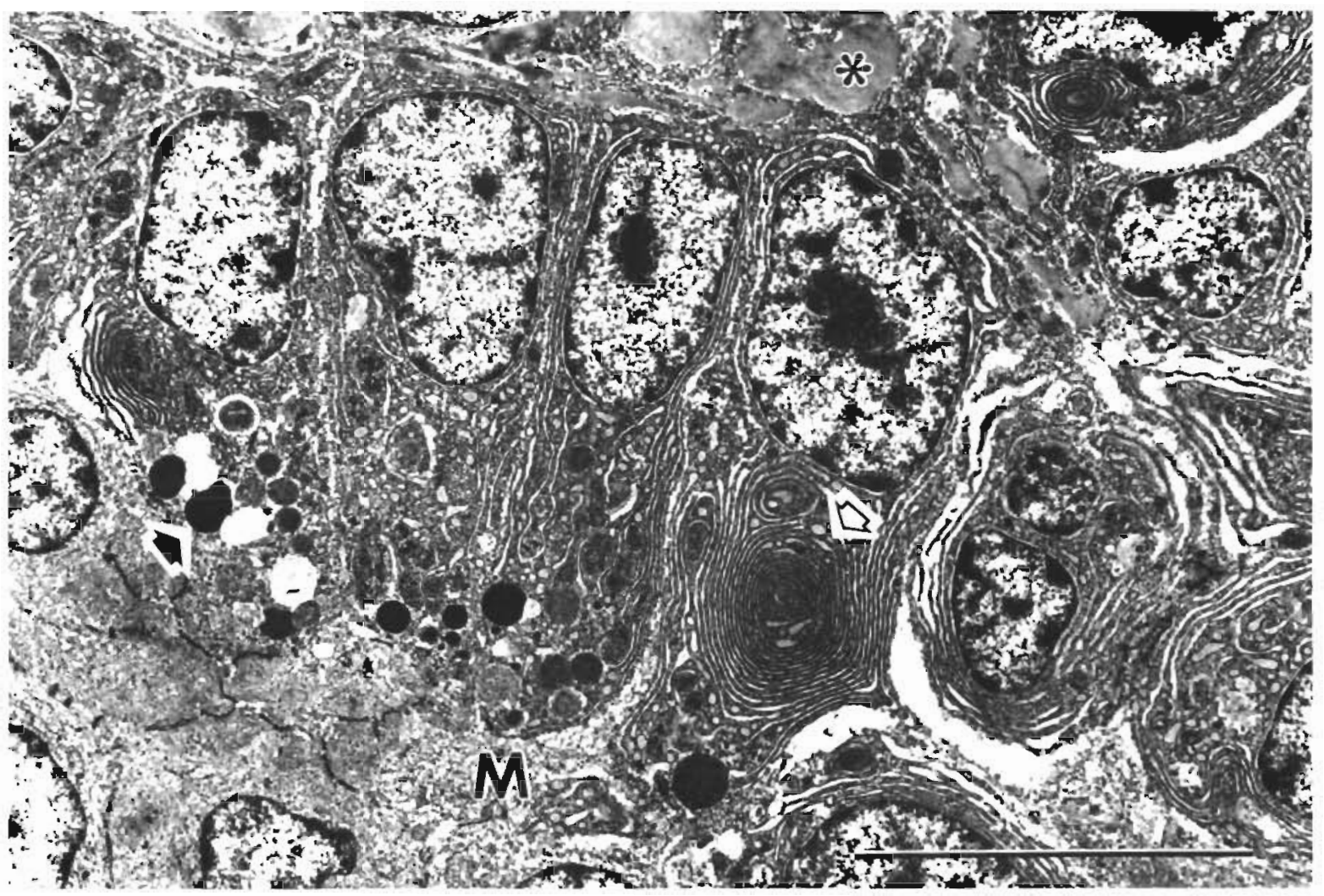

Fig. 3. Oncorhynchus kisutch. Electron micrograph of acinar cell carcinoma. Note palisading of acinar cells along fragmented basement membrane (*). Prominent endoplasmic reticulum (open arrow) is situated between apical zymogen granules (solid arrow) and cell nucleus. $M=$ acinar microlumen. $B a r=10 \mu \mathrm{m}$ 
and malignant neoplasms of the exocrine pancreas have been chemically induced in fish. Diethylnitrosamine (Thiyagarajah \& Grizzle 1986), methylazoxymethanol acetate (Fournie et al. 1987), and $N$-methyl$N^{\prime}$-nitro-N-nitrosoguanidine (Grizzle et al. 1988) have been shown to cause the formation of pancreatic adenomas, carcinomas, and adenocarcinomas in rivulus, guppies, and killifish. These animal models will be useful in helping to establish the pathogenesis of spontaneous pancreatic neoplasms in fish as well as other species.

Acknowledgements. The authors thank Jerry D. Hendricks and John W. Fournie for review of histologic material and electron micrographs, and Greg Wiens, Leslie Gilkey, and T. Chalmers for technical assistance.

\section{LITERATURE CITED}

di Sant'Agnese, P. A. (1991). Acinar cell carcinoma of the pancreas. Ultrastruct. Pathol. 15: 573-577

Fournie, J. W., Black, J. J., Vethaak, A. D. (1988). Exocrine pancreatic adenomas in the greater redhorse, Moxostoma valenciennesi Jordan, and in the European flounder, Platichthys flesus (L.). J. Fish Dis. 11: 445-448

Fournie, J. W., Hawkins, W E., Overstreet, R. M., Walker, W. W. (1987). Exocrine pancreatic neoplasms induced by methylazoxymethanol acetate in the guppy Poecilia reticulata. J. natn. Cancer Inst. 78: 715-725

Responsible Subject Editor: N. Peters, Hamburg, Germany
Grizzle, J. M., Putnam, M. R., Fournie, J. W., Couch, J. A (1988). Microinjection of chemical carcinogens into small fish embryos: exocrine pancreatic neoplasm in Fundulus grandis exposed to $N$-methyl- $N$-nitro- $N$-nitrosoguanidine. Dis aquat. Org. 5: 101-105

Jamieson, J. D. (1988). The exocrine pancreas and salivary glands. In: Weiss, L. (ed.) Cell and tissue biology: a textbook of histology. Urban and Schwarzenberg, Baltimore, p. 717-749

Nigrelli, R. F., Gordon, M. (1951). Spontaneous neoplasms in fishes: V. Acinar adenocarcinoma of the pancreas in a hybrid platyfish. Zoologica 36: 121-126

Popp, J. A. (1990). Tumors of the liver, gall bladder, and pancreas. In: Moulton, J. E. (ed.) Tumors of domestic animals. University of California Press, Berkeley, $p$ 436-457

Priester, W A. (1974). Data from eleven United States and Canadian colleges of veterinary medicine on pancreatic carcinoma in domestic animals. Cancer Res. 34: 1372-1375

Rockey, D. D., Gilkey, L. L., Wiens, G. D., Kaattari, S. L. (1991). Monoclonal antibody-based analysis of the Renibacterium salmoninaxum p57 protein in spawning chinook and coho salmon. J. aquat. Animal Health 3 : 23-30.

Scarpelli, D. G., Rao, M. S., Reddy, J. K. (1984). Studies of pancreatic carcinogenesis in different animal models. Environ. Hlth Perspect. 56: 219-227

Thiyagarajah, A., Grizzle, J. M. (1986). Diethylnitrosamineinduced pancreatic neoplasms in the fish Rivulus ocellatus marmoratus. J. natn. Cancer Inst. 77: 141-147

Webb, N. (1977). Acinar cell neoplasms of the exocrine pancreas. J. clin. Pathol. 30: 103-112

Manuscript first received: February 15, 1992

Revised version accepted: April 16, 1992 